Review began 12/12/2021 Review ended 12/14/2021 Published 12/20/2021

๑) Copyright 2021

Al Dhafiri et al. This is an open access article distributed under the terms of the Creative Commons Attribution License CCBY 4.0., which permits unrestricted use, distribution, and reproduction in any medium, provided the original author and source are credited.

\section{Attitude Toward Using the Triple Combination Bleaching Formula and Related Outcomes: A Cross-Sectional Study}

Mahdi Al Dhafiri ${ }^{1}$, Mohammed Almutairi ${ }^{2}$, Halal M. Alutaibi ${ }^{2}$, Hassan R. Aldandan ${ }^{2}$, Fatemah A. Albshr ${ }^{2}$ , Fatimah S. Alkhalifa 2

1. Department of Medicine, College of Medicine, King Faisal University, Al-Ahsa, SAU 2. College of Medicine, King Faisal University, Al-Ahsa, SAU

Corresponding author: Mahdi Al Dhafiri, maldhafiri@kfu.edu.sa

\section{Abstract}

\section{Introduction}

Kligman's formula is a topical triple combination consisting of hydroquinone, tretinoin, and topical corticosteroid. It has recently become widely popular among the general population for different purposes. Its improper use can lead to unsatisfactory results and unpleasant side effects.

\section{Aim}

This study aimed to assess the attitude, satisfaction, and complications related to topical usage of Kligman's formula among the general population in Saudi Arabia.

\section{Materials and methods}

A cross-sectional study was conducted among the general population of Saudi Arabia. A self-administered questionnaire was distributed among the targeted population using an online survey. The questionnaire includes socio-demographic characteristics, assessment of attitude, and satisfaction in using Kligman's formula. Data were tabulated and cleaned, and all statistical analyses were performed.

\section{Results}

A total of 292 participants met the inclusion criteria (26 males vs. 266 females) with a mean age of 26.9 (SD 7.71) years. Nearly $40 \%$ of participants showed a positive attitude in using Kligman's formula, while $46.9 \%$ were satisfied with using it. The most common reason for using Kligman's formula was to lighten the skin (55.8\%), while skin redness was the most commonly reported adverse effect. Factors associated with increased attitude and satisfaction were using Kligman's formula based on a doctor's prescription and regular follow-up with a dermatologist.

\section{Conclusion}

The general population showed an improper attitude toward using Kligman's formula. However, a better attitude and satisfaction rate can be seen among those using Kligman's formula with prescription and those who regularly visit a dermatologist.

Categories: Dermatology

Keywords: skin lightening, acne, satisfaction, attitude, kligman's formula

\section{Introduction}

Components of the widely known Kligman's formula are 0.1\% tretinoin, 0.1\% dexamethasone, 5.0\% hydroquinone, and hydrophilic ointment. Kligman's formula is an effective treatment option in treating many dermatological conditions such as melasma and postinflammatory hyperpigmentation [1]. Both postinflammatory hyperpigmentation and melasma are relatively common in Saudi Arabia since there are a high number of people in Saudi Arabia with brown skin (Fitzpatrick skin type III-V) [2,3].

Using skin-lightening products has become highly prevalent among the general population worldwide [4]. In Saudi Arabia, it has shown a prevalence of 56.2\% [5].Among many skin lightening techniques, Kligman's formula is one of the first chosen options, reflecting widespread usage. Other indications of Kligman's formula include dyspigmentation, getting rid of scars, and skin peeling/exfoliation [4,5].

Kligman's formula has been associated with many adverse events (AEs). Most common are erythema, desquamation, burning sensation, and steroid-induced telangiectasia. Less common AEs include acne/acne 


\section{Cureus}

breakouts, hyperpigmentation, pruritus, skin atrophy, perioral dermatitis, and hypertrichosis [6-8].

This study aims to assess the attitude toward using Kligman's formula and related outcomes among the general population in Saudi Arabia.

\section{Materials And Methods}

This is a cross-sectional study conducted among the general population of Saudi Arabia to assess the attitude toward using Kligman's formula and related outcomes. A total of 292 participants were included in the study. A self-administered questionnaire was distributed among the targeted population using an online survey. The questionnaire includes socio-demographic characteristics, assessment of attitude, and satisfaction in using Kligman's formula.Any individuals who are using Kligman's formula or have been using Kligman's formula among Saudi populations were included in this study. Incomplete questionnaires and participants who did not use Kligman's formula were excluded.Data were tabulated and cleaned in Microsoft Excel (Microsoft Corporation, Redmond, WA).

\section{Statistical analysis}

Data management and analysis were carried out using the Statistical Package for Social Sciences (SPSS) version 26 (IBM Corp, Armonk, NY). Descriptive statistics (mean, standard deviation, frequencies, and percentages) were used to quantify continuous and categorical study variables. A chi-square test was used to compare the level of attitude and satisfaction related to study variables. A p-value of $<0.05$ was considered statistically significant.

\section{Ethical approval}

All procedures that involved human participants were in accordance with the ethical standards of the institutional and/or national research committee and with the 1975 Helsinki Declaration and its later amendments or comparable ethical standards. The ethical approval was obtained from King Faisal University (reference number: KFU-REC-2021-OCT-EA00032).

\section{Results}

A total of 292 respondents have been included in the study with a mean age of 26.9 years. The majority of the respondents were female (91.1\%) and Saudi nationals (97.3\%). The majority were single (68.5\%) and had bachelor's degrees (71.9\%). Students (46.2\%) and unemployed (30.5\%) constituted most participants. Only $12.3 \%$ worked in the healthcare sector, and $8.6 \%$ were doctors.

The usage of Kligman's formula among participants is referred to different aims (Figure 1), and skin lightening represents the most common one. However, the most common sources of information for using Kligman's formula without a doctor's prescription were electronic social network sites (68.2\%), followed by friends (22.9\%) and websites (20.2\%). At the same time, television/radio was the least $(0.4 \%)$.

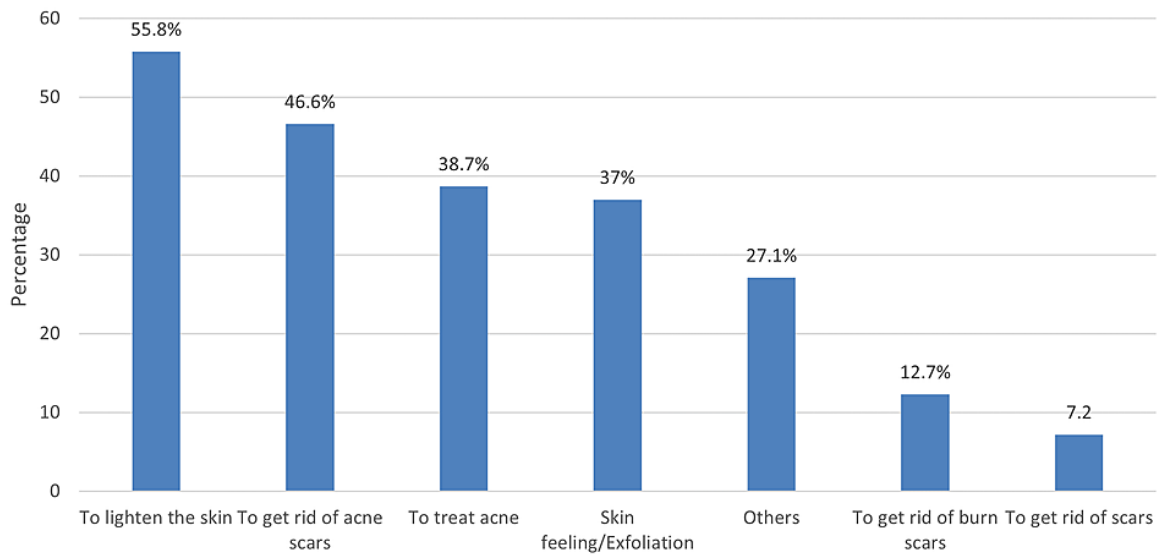

FIGURE 1: Reasons for using Kligman's formula.

Of the participants, 38\% estimated having a positive attitude in using Kligman's formula, while $23.6 \%$ reported using Kligman's formula according to doctor's prescription. The prevalence of respondents who regularly visit dermatologists to guide them regarding Kligman's formula was $17.1 \%$. More than two-thirds (67.5\%) were mixing the ingredients of Kligman's formula by themselves. Of those self-preparing Kligman's formula, $74.6 \%$ attempted to divide evenly without using any device. The proportion of respondents who bought a ready-made Kligman's formula was $35.6 \%$, while the proportion of respondents who experienced 


\section{Cureus}

side effects due to Kligman's formula was $50 \%$ (Table 1).

Study variables

N (\%)

Current attitude of using Kligman's formula

Positive

$111(38.0 \%)$

Negative

$181(62.0 \%)$

Currently using Kligman's formula based on doctor's prescription

Yes

$69(23.6 \%)$

No

$223(76.4 \%)$

Do you follow up regularly with your dermatologist to see how you use Kligman's formula?

Yes

No

$242(82.9 \%)$

How often do you use Kligman's formula?

Daily

$114(39.0 \%)$

Every 2 days

$71(24.3 \%)$

Every 3 days

$54(18.5 \%)$

Every 2 weeks

$23(07.9 \%)$

Every month

$18(06.2 \%)$

Once in a while

$12(04.1 \%)$

Timing of use for Kligman's formula

In the morning

$19(06.5 \%)$

In the evening

$235(80.5 \%)$

In the morning and evening

$38(13.0 \%)$

Duration of Kligman's formula use

Less than a month

$102(34.9 \%)$

1-2 months

$81(27.7 \%)$

2-3 months

$46(15.8 \%)$

3-6 months

$22(07.5 \%)$

6-9 months

$04(01.4 \%)$

9-12 months

More than a year

$32(11.0 \%)$

Did you collect and mix the ingredients for Kligman's formula yourself?

Yes

$197(67.5 \%)$

No

How did you divide each product (what is the percentage of that product)? $(n=197)$

Tried to divide it evenly using the scale

$22(11.2 \%)$

Tried to divide it evenly without using the scale and without any device

$147(74.6 \%)$

Divide them differently

$28(14.2 \%)$

Do you buy Kligman's formula ready-made?

Yes

$104(35.6 \%)$ 


\section{Cureus}

Experience any side effects after using Kligman's formula?

Yes

TABLE 1: Assessment of attitude toward the use of Kligman's formula ( $n=292)$.

Respondents cited face as the most common body part where Kligman’s formula was applied (92.1\%), followed by hand (14.7\%) and knees (14.7\%). It was further observed that the most frequently used ingredients were hydroquinone cream (Hiquin ${ }^{\circledR}$ ) $(64.5 \%)$, followed by tretinoin cream (Acretin $\left.{ }^{\circledR}\right)(57.4 \%)$, and adapalene cream (Differin ${ }^{\circledR}$ ) (49.2\%). Moreover, the most commonly mentioned side effect of Kligman's formula was skin redness (61.6\%), followed by a burning sensation (50.7\%) (Figure 2).

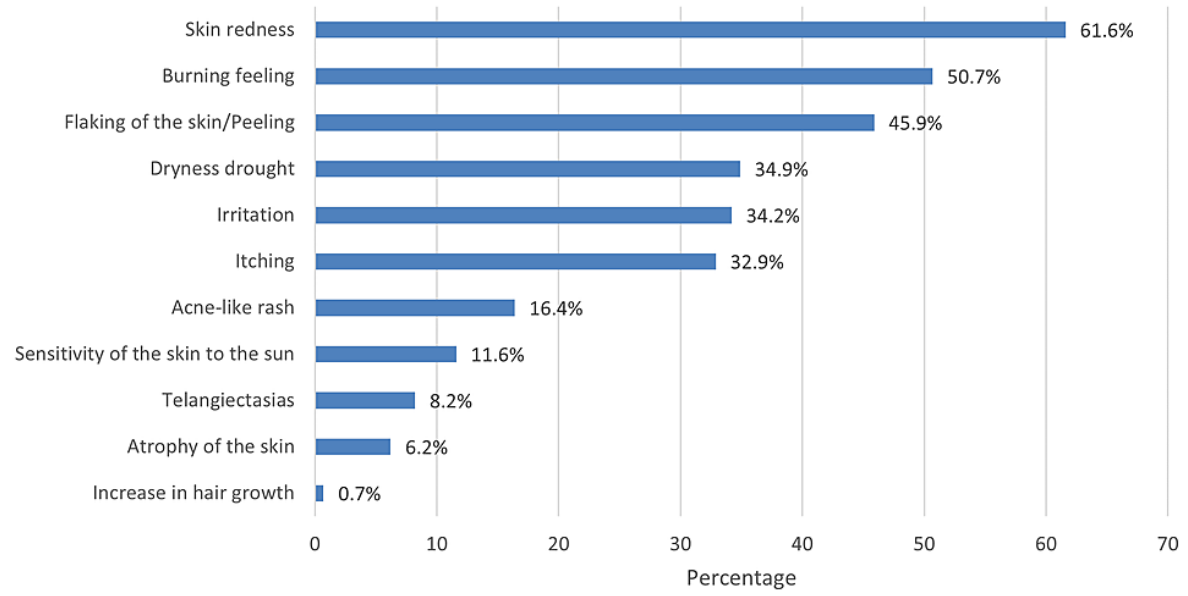

FIGURE 2: Side effects after using Kligman's formula.

The overall mean satisfaction score was 6.02 (SD 2.89) out of 10 points with $53.1 \%$, and $46.9 \%$ were classified into dissatisfied and satisfied, respectively. We also noted that $32.5 \%$ were still using Kligman's formula even after obtaining a satisfactory result. After stopping from using Kligman's formula, $44.9 \%$ expressed that they did not use anything, while $21.6 \%$ used other cream types instead. However, for the changes noticed after stopping the formula, $34.2 \%$ stated recurrence of the main problem, and $29.5 \%$ expressed persistence of the therapeutic result (Table 2). 


\section{Cureus}

Variables

Satisfaction after using Kligman's formula (mean \pm SD)

Dissatisfied (score $\leq 6)$

Satisfied (score $>6$ )

Duration of Kligman's formula use after reaching satisfaction

Less than a month

1-3 months

3-6 months

6-12 months

I was not satisfied with the result

Other

If you stopped using Kligman's formula, what did you do after stopping?

I used another type of cream

I did not use anything

I went to the dermatologist to treat the side effects

I have not stopped yet

Other

What changes did you notice on your skin after you stopped using Kligman's formula?

Maintaining the therapeutic result

Repetition of the main problem

Persistent side effects

Worsening side effects

I have not stopped yet

TABLE 2: Satisfaction and outcome after using Kligman's formula ( $n=292)$.

When measuring the relationship of the attitude and satisfaction among participants, it was found that positive attitude in using Kligman's formula was more common among those using Kligman's formula based on doctor's prescription $\left(\mathrm{X}^{2}=20.030 ; \mathrm{p}<0.001\right)$, those who had regular follow up with dermatologist $\left(\mathrm{X}^{2}=\right.$ 17.290; $\mathrm{p}$ <.001), and those who bought ready-made Kligman's formula $\left(X^{2}=5.679 ; \mathrm{p}=0.017\right)$, while negative attitude was more common among those who were using self-made Kligman's formula $\left(\mathrm{X}^{2}=4.119\right.$; $\mathrm{p}=0.042)$.

\section{Discussion}

The present study attempted to evaluate the attitude and satisfaction in using Kligman's formula while highlighting the adverse effect of misusage. The findings of this study revealed that only $38 \%$ exhibited a positive attitude and the rest (62\%) had a negative attitude about it. Of those using Kligman's formula, 26\% were using it according to the doctor's advice, with $17.1 \%$ of them having a follow-up visit with the dermatologist. In India [9], a study revealed a significant increase in the awareness of the long-term use of topical steroids, including Kligman's formula, with $55.2 \%$ of the medical students using it without prescriptions. The use of topical steroids without doctor's prescriptions had been well-discussed in most publications. For instance, Jha et al. [10] reported that $42.9 \%$ of the patients bought topical corticosteroids (TC) containing creams over the counter without prescription, among them, $20 \%$ were recommended by their friends, family members, and neighbors, and $8.5 \%$ were recommended by a beautician. These findings corroborated the report of Sendrasoa et al. [11], which indicated that the majority of respondents (61\%) 
obtained TC from cosmetic retailers, pharmacy stores (23\%), and beauticians (12\%), with only $0.26 \%$ using TC based on the physician's prescription.

Alrayyes et al. [5] noted that skin lightening products could harm the skin since users were unaware of the product's active ingredients. Incidentally, in this study, due to the inappropriate use of Kligman's formula, half of the respondents had experienced adverse effects, including $4.5 \%$ reported photosensitivity of the face when exposed to sunlight. These results are in accordance with the study of Majid [8], indicating that complaints of side effects due to the Kligman's formula were seen among $26 \%$ of the patients. In a study conducted by Sendrasoa et al. [11], 13\% of the Madagascar respondents planned to seek dermatological care due to cutaneous adverse effects after using TC. However, most of them were hesitant to proceed due to costly services.

Moreover, adverse reactions resulting from using the TC or combination therapy, including Kligman's formula, may vary according to the type of cream or containing ingredients. It includes acne, hypopigmentation, pigmentation disorder, and cutaneous atrophy $[10,11]$. In our study, skin redness, burning feeling, and skin peeling were the most common side effects experienced by the respondents after using Kligman's formula. While respondents in this study indicated adverse reactions when using the formula. However, nearly three-quarters (72.9\%) were using sunscreen to reduce the side effect of the mixed ingredients or to protect against the dyspigmentation of the skin.

Siadat et al. [12] pointed out that combining modified Kligman's cream + intense pulsed light yielded better satisfaction rates than modified Kligman's alone. They further surmised that the combination showed better efficacy and faster response in the treatment of the post-burn hyperpigmentation without experiencing side effects after the treatment. Adverse reactions due to the application of the combined formula may vary according to the conditions to be treated. Therefore, it is essential to consult a doctor before using it.

The overuse of Kligman's formula has also been noticed in this study. In our observation, about one-third (32.1\%) of the respondents were still using the combination therapy even after their satisfaction. Some indicated that it is necessary to maintain therapeutic results, while some of them used another type of cream after the discontinuation of Kligman's formula. Moreover, more than one-third (34.2\%) complained of conditions recurrence, which could be the main reason for the extended usage of the combined therapy. Similarly, Dhanalakshmi et al. [9] found that students used the topical medication beyond the prescribed period. A total of $52.6 \%$ had used the medication for a maximum of one month, and the most common reasons for continuation as cited by the medical students were the treatment had no effect (30\%) or skin glowed after the application (23.3\%).

The limitations of the present study include the sample size and self-reported attitude and side effects. In addition, the correlations are measured using a cross-sectional design, which may not provide a wellestablished casualty. Nevertheless, this study could be used as a baseline to further investigate this problem in the future.

\section{Conclusions}

Kligman's formula represents one of the therapeutic options for certain skin conditions, especially melasma and post-inflammatory hyperpigmentation. However, its application and preparation under medical advice with regular follow-up are crucial. Improper and uncontrolled prolonged formula usage could lead to different cutaneous side effects, including skin atrophy, acne, and rosacea.

\section{Additional Information \\ Disclosures}

Human subjects: Consent was obtained or waived by all participants in this study. Research Ethics Committee, King Faisal University issued approval KFU-REC-2021-OCT-EA00032. Having reviewed the details submitted by the applicant regarding the above-named research project, the Research Ethics Committee at King Faisal University grants its ethical approval to the protocol. Projects may be subject to an audit or any other form of monitoring by the committee at any time. The committee may request a regular report on the progress of the project to ensure that researchers are committed to the highest ethical standards. Researchers are held accountable for the storage, retention, and security of original data obtained from projects. Any substantial alterations to the project or emerging events or matters that may affect the ethical acceptability of the project must be reported immediately to the committee via email (ialjreesh@kfu.edu.sa) or phone (0096615899773). Animal subjects: All authors have confirmed that this study did not involve animal subjects or tissue. Conflicts of interest: In compliance with the ICMJE uniform disclosure form, all authors declare the following: Payment/services info: All authors have declared that no financial support was received from any organization for the submitted work. Financial relationships: All authors have declared that they have no financial relationships at present or within the previous three years with any organizations that might have an interest in the submitted work. Other relationships: All authors have declared that there are no other relationships or activities that could appear to have influenced the submitted work. 


\section{Cureus}

\section{References}

1. Kligman AM, Willis I: A new formula for depigmenting human skin . Arch Dermatol. 1975, 111:40-8.

2. Alakloby OM: Pattern of skin diseases in Eastern Saudi Arabia . Saudi Med J. 2005, 26:1607-10

3. Abanmi A, Al-Enezi M, Al Hammadi A, Galadari I, Kibbi AG, Zimmo S: Survey of acne-related postinflammatory hyperpigmentation in the Middle East. J Dermatolog Treat. 2019, 30:578-81. 10.1080/09546634.2018.1542807

4. Alrayyes SF, Alrayyes SF, Farooq Dar U: Skin-lightening practices behind the veil: an epidemiological study among Saudi women. J Cosmet Dermatol. 2020, 19:147-53. 10.1111/jocd.12972

5. Alrayyes SF, Alrayyes SF, Farooq UD: Skin-lightening patterns among female students: a cross-sectional study in Saudi Arabia. Int J Womens Dermatol. 2019, 5:246-50. 10.1016/j.ijwd.2019.04.026

6. Torok HM, Jones T, Rich P, Smith S, Tschen E: Hydroquinone $4 \%$, tretinoin $0.05 \%$, fluocinolone acetonide 0.01\%: a safe and efficacious 12-month treatment for melasma. Cutis. 2005, 75:57-62.

7. Sarkar SK, Sen KG, Mostofa MK, Das AR, Saha SK, Islam MS: The role of triple combination topical agents in the treatment of facial melasma. Faridpur Med Coll J. 2017, 12:68-70.

8. Majid I: Mometasone-based triple combination therapy in melasma: is it really safe? . Indian J Dermatol. 2010, 55:359-62. 10.4103/0019-5154.74545

9. Dhanalakshmi K, Pious MT, Sudarvizhi A, Jennifer G: Awareness and attitude of medical students over the misuse of topical steroids- prospective study in 3rd year medical students in a medical college, Tamil Nadu. IP Indian J Clin Exp Dermatol. 2021, 7:115-9. 10.18231/j.ijced.2021.022

10. Jha AK, Sinha R, Prasad S: Misuse of topical corticosteroids on the face: a cross-sectional study among dermatology outpatients. Indian Dermatol Online J. 2016, 7:259-63. 10.4103/2229-5178.185492

11. Sendrasoa FA, Ranaivo IM, Andrianarison M, Raharolahy O, Razanakoto NH, Ramarozatovo LS, Rapelanoro Rabenja F: Misuse of topical corticosteroids for cosmetic purpose in Antananarivo, Madagascar . Biomed Res Int. 2017, 2017:9637083. 10.1155/2017/9637083

12. Siadat AH, Iraji F, Bahrami R, et al.: The comparison between modified Kligman formulation versus Kligman formulation and intense pulsed light in the treatment of the post-burn hyperpigmentation. Adv Biomed Res. 2016, 5:125. 10.4103/2277-9175.186997 